\title{
Percutaneous Stone Removal in an Adult with a Continent Cutaneous Reservoir: Case Report and Review of the Literature
}

\author{
Bhalaajee Meenakshi-Sundaram, MD, Jonathan A. Jensen, MD, Jared P. Higley, MD, \\ Caitlin T. Coco, MD, and Jonathan E. Heinlen, MD
}

\begin{abstract}
Stone formation is a known long-term complications of continent urinary reservoirs. We present a rare case of a large stone in a continent cutaneous reservoir in a 56-year-old male managed with percutaneous cystolithotomy. The patient presented with recurrent urinary tract infections and stomal incontinence. CT revealed an 8-cm stone occupying the entire lumen of his reservoir. Rather than removing this large stone through an open approach, the pouch was accessed percutaneously and lithotripsy was performed. In this report, we demonstrate our operative technique and that percutaneous cystolithalopaxy may be a safe and effective alternative for the management of large stones within continent cutaneous reservoirs.
\end{abstract}

Keywords: Kock Pouch, cystolithalopaxy, reservoir, stone

\section{Introduction}

A LTHOUGH THE PERCUTANEOUS APPROACH to reservoir stones has been well documented in pediatric patients, fewer reports exist in adults. Furthermore, the endoscopic management of reservoir stones in adults has generally been reserved for smaller stones and access is obtained through the efferent limb of the reservoir or through a catheterizable stoma. For larger stones, an open approach has been favored over percutaneous access because of the risk of injury to adjacent structures in patients who have had complex reconstructive procedures. We present a case of percutaneous management of a large stone in a patient with a Kock Pouch and a history of multiple abdominal procedures.

\section{Case Report}

A 56-year-old male with a history of myelomeningocele (MM) presented to clinic with abdominal pain, recurrent urinary tract infections (UTIs), and stomal incontinence. He underwent creation of an ileal conduit at age 5. Owing to recurrent anastomotic strictures and a desire for continence, he subsequently underwent creation of a Kock Pouch at age 25. Since that time, he has performed clean intermittent catheterization four to six times daily but has never irrigated his pouch to clear mucous and debris. As a result, he developed multiple small stones that were treated with laser lithotripsy through the catheterizable limb of his pouch. The previous stones were noted to arise from the staple line within the pouch.

On physical examination, a healed midline laparotomy incision, the previous urostomy scar in the right upper quadrant, and a catheterizable stoma in the right lower quadrant were noted. X-ray of the abdomen and pelvis revealed an $8 \mathrm{~cm}$ stone within the pelvis (Fig. 1). On CT, the stone was noted to occupy the entire lumen of the reservoir. The pouch itself was noted to extend under the midline scar and umbilicus, but no bowel was noted between the rectus fascia and the pouch (Fig. 2). Given this finding, a percutaneous approach to the stone was selected to spare the patient the potential morbidity and extended recovery associated with an open abdominal procedure.

In the operating room, flexible cystoscopy of the pouch was performed through the catheterizable stoma. The large stone was located dependently and multiple smaller fragments were also noted to be scattered throughout the pouch. A spinal needle was placed percutaneously into the pouch and urine was aspirated to define our percutaneous tract into the pouch. Next, the skin surrounding the needle was incised and a $12 \mathrm{~mm}$ laparoscopic trocar was placed into the pouch.

Department of Urology, OU Medical Center, Oklahoma City, Oklahoma.

(C) Bhalaajee Meenakshi-Sundaram et al. 2017; Published by Mary Ann Liebert, Inc. This is an Open Access article distributed under the terms of the Creative Commons Attribution License, which permits unrestricted use, distribution, and reproduction in any medium, provided the original work is properly cited. 


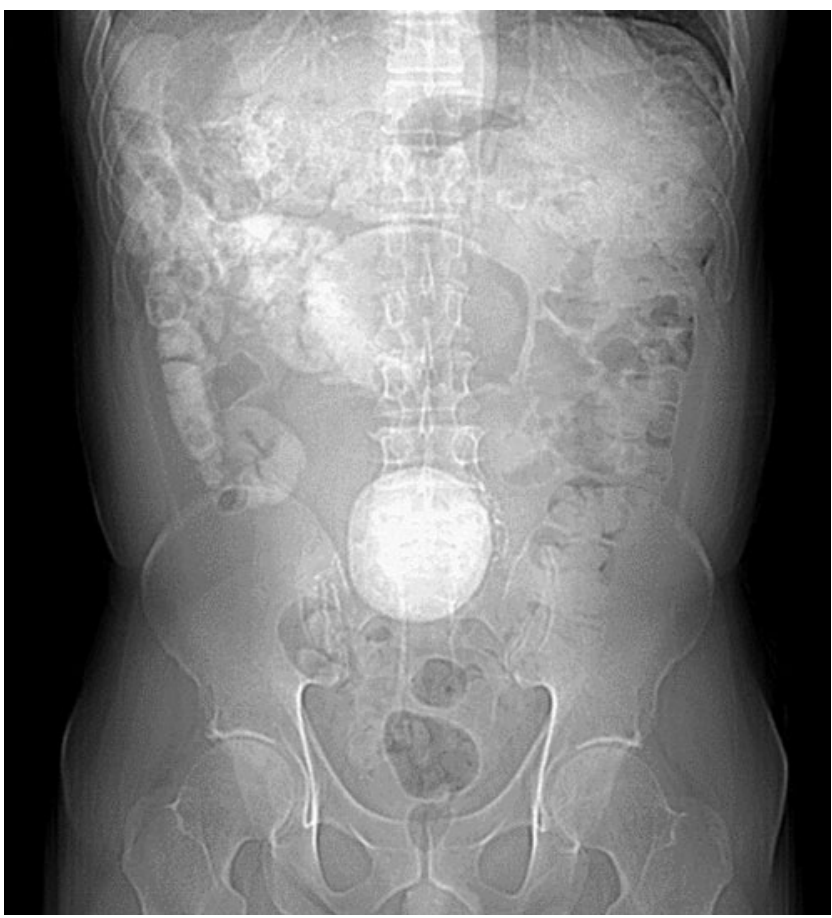

FIG. 1. X-ray demonstrating stone within cutaneous reservoir.

The flexible cystoscope was left in the catheterizable stoma to observe entry of the trocar into the pouch. An Inzii 12/15 mm endoscopic retrieval bag was then placed through the laparoscopic trocar and the stone was collected. After removal of the trocar, the neck of the bag was brought through the incision and a rigid nephroscope and Olympus CyberWand Ultrasonic Lithotriptor were placed into the bag to perform lithotripsy. Once a sufficient number of fragments had been

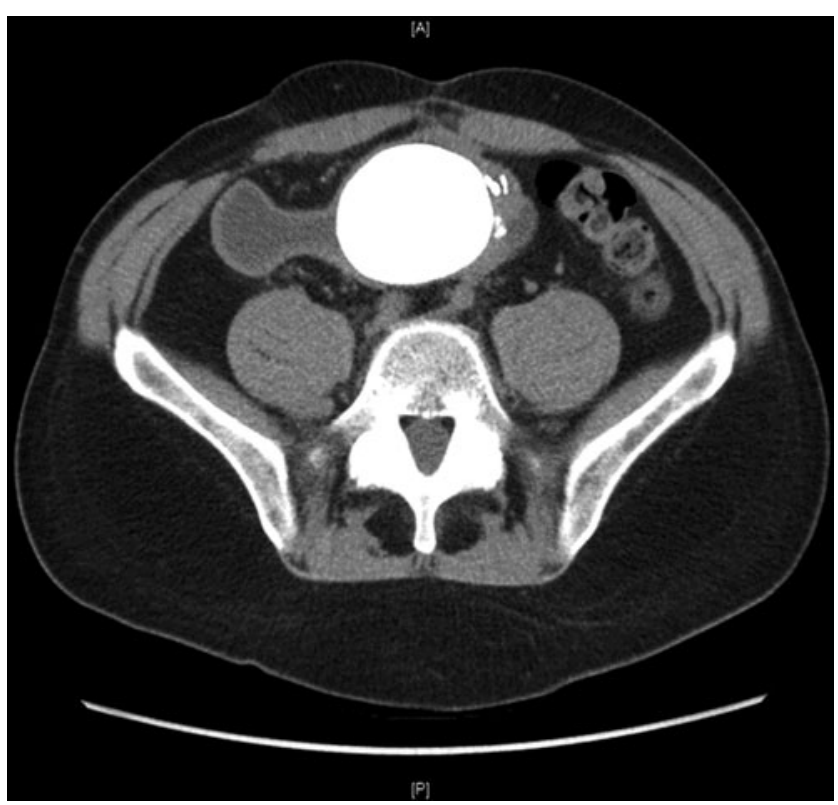

FIG. 2. Axial CT image of stone occupying entire lumen of reservoir.

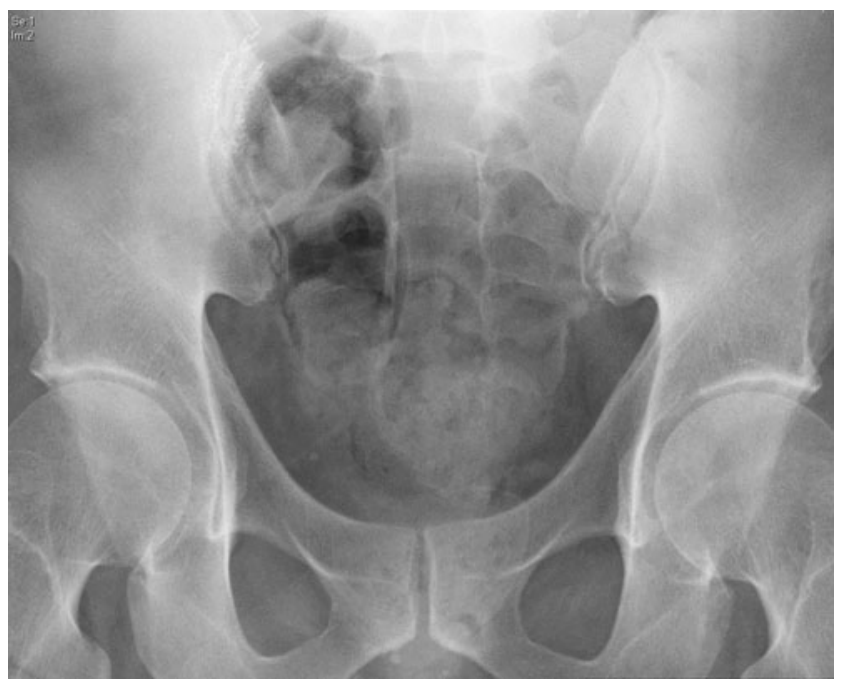

FIG. 3. Postoperative X-ray demonstrating stone clearance.

aspirated, the retrieval bag and remaining stone fragments were pulled through the laparoscopic port site. Repeat endoscopy of the pouch revealed clearance of the stone and no injury to the pouch itself. The fascia at the port site was closed with 2-0 Vicryl and the skin was closed with 4-0 Monocryl. An $18 \mathrm{~F}$ catheter was left in the catheterizable stoma.

The patient was discharged home on postoperative day 1 . An X-ray done 2 weeks postoperatively showed no residual stone (Fig. 3). The Foley catheter was also removed at this time. The stone composition was found to be $55 \%$ struvite and $45 \%$ calcium phosphate. The patient was started on a regimen of twice daily saline irrigation of the pouch in addition to regular catheterization and has not developed any more stones.

\section{Discussion}

The formation of bladder stones is a common complication after continent urinary diversions with incidences as high as $30 \%-50 \%$ depending upon the type of diversion. ${ }^{1}$ Numerous factors including metabolic abnormalities, urinary stasis, mucous production, presence of foreign bodies, and UTIs secondary to urease-producing organisms all promote urolithiasis within continent diversions. ${ }^{2}$ Large calculi within continent diversions pose significant challenges with regard to approach as it is important to completely retrieve all fragments. Residual fragments may serve as niduses for infection and further stone formation. Although shock wave and laser lithotripsy may be suitable for smaller stones, they are less effective in treating larger stone burdens. Small stoma size precludes the passage of large fragments or nephroscopes necessary for treating large stones. ${ }^{3}$

Previously, open cystolithotomy had been advocated as the standard of care for large stones because of the necessity for removing the entirety of the stone burden within urinary diversions. ${ }^{4}$ However, multiple series in the pediatric population have demonstrated equivalent effectiveness for the percutaneous approach. ${ }^{2}$ The utilization of a retrieval bag helps reduce the residual stone burden by preventing migration of the stone fragments during ultrasonic lithotripsy. 
Similar series are not present in adult patients with Kock Pouches and only anecdotal reports regarding management of pouch stones are available. In our patient, open cystolithotomy may have been appropriate given the large stone burden, but the endoscopic approach carried a lower risk of urine leak and resulted in a shorter postoperative hospital stay.

\section{Conclusions}

Percutaneous removal of stones from continent cutaneous reservoirs is a safe and effective alternative to open cystolithotomy in adults with large stone burdens. We present a rare case of percutaneous cystolithotomy for a large stone in an adult who previously underwent Kock Pouch creation for management of neurogenic bladder secondary to MM. To our knowledge, this is one of very few similar reports available in the literature.

\section{Disclosure Statement}

No competing financial interests exist.

\section{References}

1. Khoury AE, et al. Stone formation after augmentation cystoplasty: The role of intestinal mucus. J Urol 1997;158(3 Pt 2): 1133-1137.

2. Lam PN, et al. Percutaneous cystolithotomy of large urinarydiversion calculi using a combination of laparoscopic and endourologic techniques. J Endourol 2007;21:155-157.
3. Jarrett TW, et al. Stone entrapment during percutaneous removal of infectious stones from a continent diversion. J Urol 1999;162(3 Pt 1):775-776.

4. Blyth B, et al. Lithogenic properties of enterocystoplasty. J Urol 1992;148(2 Pt 2):575-577.

Cite this article as: Meenakshi-Sundaram B, Jensen JA, Higley JP, Coco CT, Heinlen JE (2017) Percutaneous stone removal in an adult with a continent cutaneous reservoir: case report and review of the literature, Journal of Endourology Case Reports 3:1, 179-181, DOI: 10.1089/cren.2017.0073.

Address correspondence to: Bhalaajee Meenakshi-Sundaram, MD

Department of Urology

OU Medical Center

920 Stanton L. Young Boulevard, WP 3150

Oklahoma City, OK 73072

E-mail: bhalaajee-meenaksi-sundaram@ouhsc.edu

$$
\begin{aligned}
& \text { Abbreviations Used } \\
& \text { CT }=\text { computed tomography } \\
& \text { MM }=\text { myelomeningocele } \\
& \text { UTI }=\text { urinary tract infection }
\end{aligned}
$$

